\title{
DIMENSIONES PEDAGÓGICAS QUE CONFIGURAN LAS PRÁCTICAS DE APRENDIZAJE-SERVICIO
}

\section{Pedagogical Dimensions that Configure Service-Learning Practices}

\author{
DOMINGO MAYOR PAREDES*1 \\ http://orcid.org/0000-0002-4285-4210 \\ *Universidad de Almería, España. Correspondencia: dmp810@ual.es
}

Recibido: 22-07-2018

Revisado: 30-10-2018

Aceptado: 01-05-2019

\begin{abstract}
Resumen: Este artículo tiene como propósito enfocar en las diversas dimensiones pedagógicas y sociales que vertebran los proyectos de aprendizaje-servicio, un objeto de estudio de naturaleza multidimensional e interdisciplinar. A partir de la disección de los planteamientos de autores reconocidos en el ámbito iberoamericano, se ofrece una visión panorámica de cada una de las regularidades: aprendizaje, servicio, participación, reflexión, intencionalidad educativa, entre otras dimensiones que van tejiendo los proyectos. Estas regularidades sirven para identificar las señas de identidad de dichas prácticas, así como las similitudes y diferencias que mantienen con otras prácticas experienciales y con otros métodos educativos.
\end{abstract}

Palabras clave: aprendizaje-servicio; práctica pedagógica; relación escuela-comunidad; participación de los estudiantes; innovación pedagógica.

Abstract: The purpose of this article is to focus on the various pedagogical and social dimensions that vertebrate service-learning projects, a multidimensional and interdisciplinary subject of study. From the dissection of the approaches of recognized authors in the Ibero-American field, a panoramic vision of each of the regularities is offered: learning, service, participation, reflection, educational intentionality, among other dimensions that are woven together by the projects. These regularities serve to identify the signs of identity of these practices, as well as the similarities and differences they maintain with other experiential practices and with other educational methods.

Keywords: service-learning, educational practice, school-community relationship, student participation, educational innovation.

\footnotetext{
${ }^{1}$ Máster en Políticas y Prácticas de Innovación Educativa, Universidad de Almería (España). Doctorado en Educación por la Universidad de Almería. Líneas de investigación: aprendizaje-servicio, educación social y desarrollo comunitario.
} 


\section{EL APRENDIZAJE-SERVICIO, UNA METODOLOGÍA INNOVADORA MEDIADA POR UNA DOBLE DIMENSIÓN: PEDAGÓGICA Y SOCIAL}

El significante aprendizaje-servicio (ApS), en su devenir sociohistórico, ha sido interpretado y materializado de múltiples formas, como lo evidencian las diversas definiciones y prácticas halladas. Un estudio realizado por Kendal (citado en MartínezOdria, 2008, p. 629) encontró hasta 147 nociones diferentes en la literatura especializada para significar esta práctica educativa de naturaleza multidimensional (instituciones que intervienen, ámbitos donde se desarrolla, dimensiones pedagógicas que la estructuran, tipología de los servicios, edades de los participantes, etcétera) y multidisciplinar (pedagogía, psicología, sociología) que se viene realizando por múltiples agencias en distintos contextos socioculturales. Ello supone que hoy siga habiendo dificultades para encerrar esta práctica educativa bajo una misma definición.

De las múltiples definiciones que existen, nos vamos a centrar en algunas propuestas presentadas por algunos especialistas y una institución pública que son reconocidos como impulsores del ApS, tanto en sus respectivos países como en el ámbito internacional. Para Furco (2011, p. 23), el ApS es "una pedagogía de enseñanza por la que los estudiantes adquieren una mejor comprensión del contenido académico aplicando competencias y conocimientos al beneficio de la sociedad". Para Puig y Palos (2006, p. 4), es "una propuesta educativa que combina procesos de aprendizaje y de servicio a la comunidad en un solo proyecto bien articulado en el que los participantes se forman al trabajar sobre necesidades reales del entorno con el objetivo de mejorarlo".

Por su parte, el Ministerio de Educación de Argentina (Tapia, González y Elicegui, 2005 , p. 10) plantea que el ApS es "un servicio protagonizado por los estudiantes, destinado a atender necesidades reales y efectivamente sentidas de una comunidad, planificado institucionalmente de forma integrada con el currículo, en función del aprendizaje de los estudiantes".

Teniendo en cuenta las mencionadas definiciones y los trabajos realizados por Martínez-Odria (2008), Puig (2009) y Tapia et al. (2005), quienes han analizado diversas experiencias así como distintas descripciones y aproximaciones teóricas, se observan, por un lado, algunas diferencias relacionadas con el protagonismo de los participantes o el nombre asignado a esta práctica educativa y, por otro, diversas dimensiones que tienden a repetirse en todas ellas: actividad educativa intencionalmente planificada, interdependencia entre los aprendizajes curriculares y el servicio a la comunidad, propósito de responder a demandas sociales y participación fuerte de los protagonistas.

Cabe mencionar que el estudio científico de la evolución de este fenómeno ha posibilitado "un consenso a nivel internacional que apunta a definir al ApS a partir de tres rasgos fundamentales" (Montes, Tapia y Yaber, 2011, p. 9):

- Servicio a la comunidad con el propósito de dar respuesta a necesidades reales y sentidas. 
- Protagonismo de los y las participantes (estudiantado de distintos niveles educativos, docentes, representantes de entidades sociales y recursos públicos).

- Planificación intencional e integrada de los contenidos curriculares y las actividades que conforman el servicio a la comunidad.

\section{Características del aprendizaje-servicio como práctica pedagógica}

En el contexto iberoamericano existe un acuerdo generalizado para identificar cinco dimensiones que configuran la arquitectura pedagógica del ApS: aprendizaje, servicio, actividad de utilidad social, participación y reflexión (Batlle, 2013; Díaz, 2006; Puig, 2015; Montes et al., 2011). Para adentrarnos en ellas, hemos seguido los trabajos de Puig, Batlle, Bosch y Palos (2007) y Puig (2015), ya que incluyen algunas características más para dar cuenta, entre otros asuntos, de la colaboración entre la escuela y la comunidad, a partir de la conformación de una red de partenaires. ${ }^{2}$ El aprendizaje-servicio, entonces, tiene las siguientes características:
Un proyecto educativo con utilidad social.
Un método para la educación formal y no formal, para todas las edades y
que debe contar con un espacio temporal preciso.
Un servicio para aprender y colaborar en un marco de reciprocidad.
Un proceso de adquisición de conocimientos y competencias para la vida.
Un método de pedagogía activa que precisa de un educador más que de
un enseñante.
Una red de partenaires y de instancias de conexión y apoyo.
Un impacto formativo y transformador múltiple (Puig et al., 2007, p. 55).

Estas dimensiones no siempre se manifiestan de la misma manera ni con el mismo énfasis, pero "expresan un ideal educativo o notas deseables al que cada experiencia concreta, como realidad singular, se va aproximando poco a poco o sería deseable que así lo hiciera" (Puig y Palos, 2006, p. 9).

\section{Aprendizaje-servicio y otras prácticas educativas experienciales}

El reconocimiento de las regularidades que sostienen al ApS como práctica educativa permiten, por un lado, dotarla de identidad como objeto de estudio científico y realizar investigaciones sobre los impactos que produce (Furco, 2011) y, por otro, diferenciarla de otras prácticas educativas experienciales que se vienen desarrollando en el ámbito comunitario. En esta línea, la Universidad de Stanford creó los cuadrantes de aprendizaje-servicio (Figura 1) para representar la relación entre el aprendizaje y el servicio en distintas prácticas experienciales (Tapia et al., 2005). Este instrumento es

\footnotetext{
${ }^{2}$ El partenariado es un proceso relacional donde dos o más organizaciones se unen para crear algo que por sí solas, no podrían conseguirlo (Puig et al., 2007).
} 
muy utilizado en la bibliografía especializada para diferenciar el ApS de otras actividades similares con las que suele confundirse (Luna, 2010).

Figura 1: Cuadrantes de aprendizaje-servicio del Service-learning Center de la Universidad de Standford

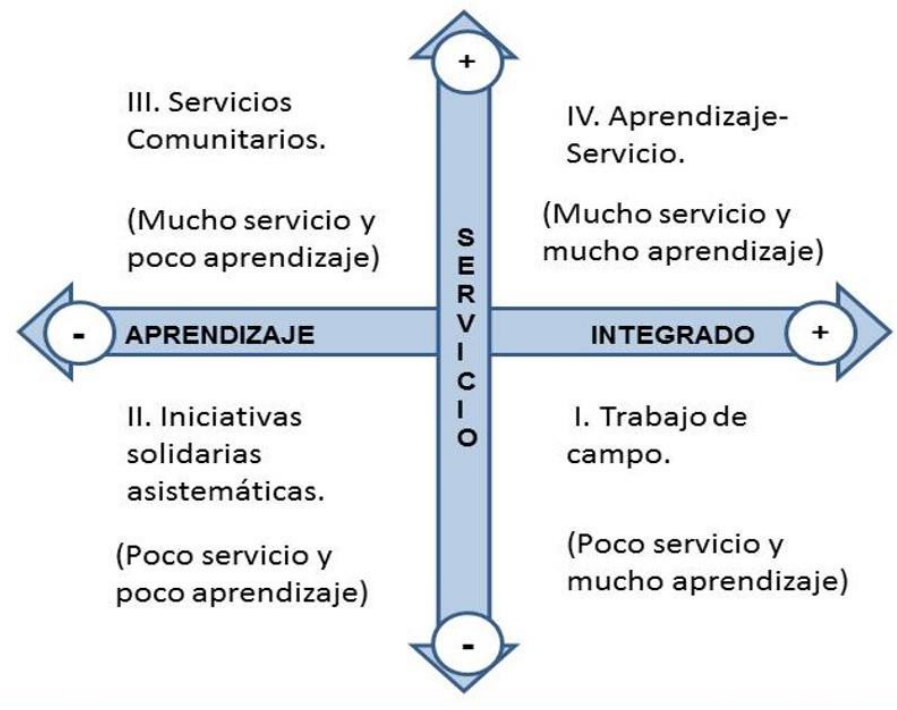

Fuente: Elaboración propia a partir de Montes, Tapia y Yaber (2011, p. 15)

Siguiendo la figura, el eje vertical indica la mayor o menor calidad del servicio que se presta a la comunidad y el eje horizontal se refiere al nivel de integración de los aprendizajes académicos con el servicio desarrollado. En función de los ejes se delimitan los cuatro tipos de prácticas educativas experienciales. Su reconocimiento posibilita su clarificación conceptual y los pasos a seguir para convertir cada una de esas prácticas experienciales en una propuesta de ApS (Montes, Tapia y Yaber, 2011):

1) Los trabajos de campo, al igual que otros métodos de análisis de la realidad (aprendizaje por problemas, estudio de casos, etc.), se enfocan en que el alumnado aprenda involucrándose en la realidad de sus comunidades, pero considerándolas, fundamentalmente, como objeto de estudio. Esto permite al estudiantado adquirir conocimientos y habilidades, pero no existe la intencionalidad educativa de transformar las comunidades. Un ejemplo podría ser cuando el alumnado realiza el análisis de un río en malas condiciones de salubridad, con el propósito de medir diversas características de algunas propiedades del agua.

2) El servicio comunitario, al contrario que las prácticas de trabajo de campo focalizadas en los aprendizajes, busca incidir en algunos elementos de la realidad, por lo que existe una decisión intencionada de promover valores (solidaridad, igualdad, etc.) y desarrollar, 
en los participantes, actitudes de compromiso social y de participación ciudadana. Esta acción puede resultar un servicio de alta calidad o una estrategia efectiva de educación en valores, pero, en general, ese aprendizaje discurre en paralelo con la formación académica sin enriquecerse mutuamente. Siguiendo con el ejemplo del río, un grupo de estudiantes participan como voluntarios en una ONG u otro tipo de institución en un servicio encargado de la limpieza y reforestación de los alrededores del río, actividad que se implementa durante el curso escolar.

3) Las iniciativas solidarias asistemáticas están constituidas por actuaciones puntuales que se implementan desconectadas de los contenidos curriculares. Son asistemáticas porque no están planificadas intencionalmente, por lo que no garantizan una implicación real en el servicio ni constituyen una fuente de adquisición de aprendizajes. En estas acciones la comunidad es el principal destinatario. El ejemplo aquí podría ser la asistencia del alumnado a un acto organizado por una institución para colaborar en la limpieza de los alrededores de un río.

4) Las prácticas de ApS están caracterizadas por la planificación intencional de los contenidos formativos y las actividades que configuran el servicio que se va a implementar en la comunidad, con el propósito de mejorar alguna dimensión de esta. Dichos elementos estructurales conforman un círculo virtuoso (Figura 2) ya que, por una parte, los aprendizajes obtenidos en el proceso formativo mejoran la calidad del servicio y, por otra, la realización del servicio estimula la adquisición o producción de nuevos conocimientos para resolver adecuadamente la necesidad detectada (Batlle, 2010; Montes, Tapia y Yaber, 2011).

Los participantes aquí adoptan un rol protagónico en las distintas fases de realización del proyecto: diseño, implementación y evaluación. Volviendo al ejemplo del río, el estudiantado o grupo en formación, junto con el maestro-profesor-educador, deciden investigar las causas de la contaminación del río en el marco de distintas materias curriculares o formativas (biología, química, ciencias de la naturaleza, campaña de sensibilización, etc.) con la intencionalidad de diseñar una campaña informativa dirigida a la población local para mostrar los hallazgos. Para realizar el proyecto, cuentan con la colaboración de un grupo ecologista y de una asociación de vecinos de la zona. 
Figura 2. Círculo virtuoso

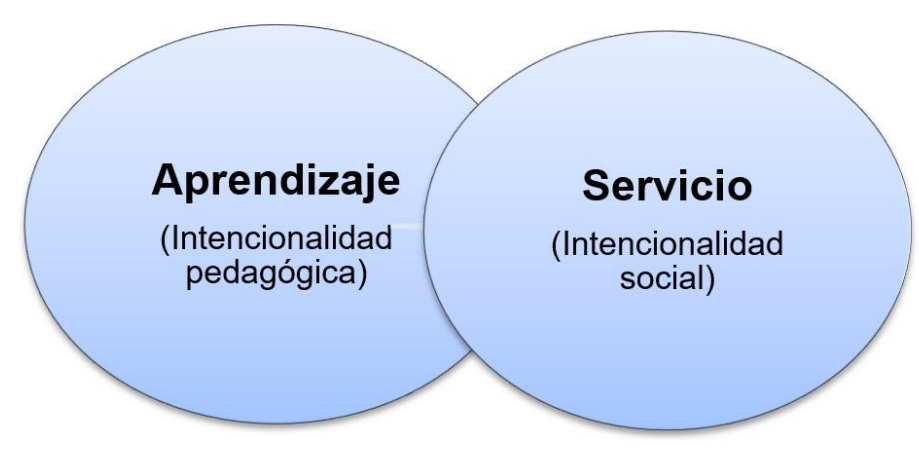

Fuente: Elaboración propia

a partir de Montes, Tapia y Yaber (2011).

Las investigaciones realizadas en los últimos años, junto a la profusión de proyectos de ApS y su difusión en el ámbito internacional, ha posibilitado distinguir grandes campos de prácticas educativas experienciales, así como los pasos necesarios para favorecer el tránsito hacia la formulación de un proyecto de ApS (Figura 3).

Figura 3. Las transiciones hacia el aprendizaje-servicio

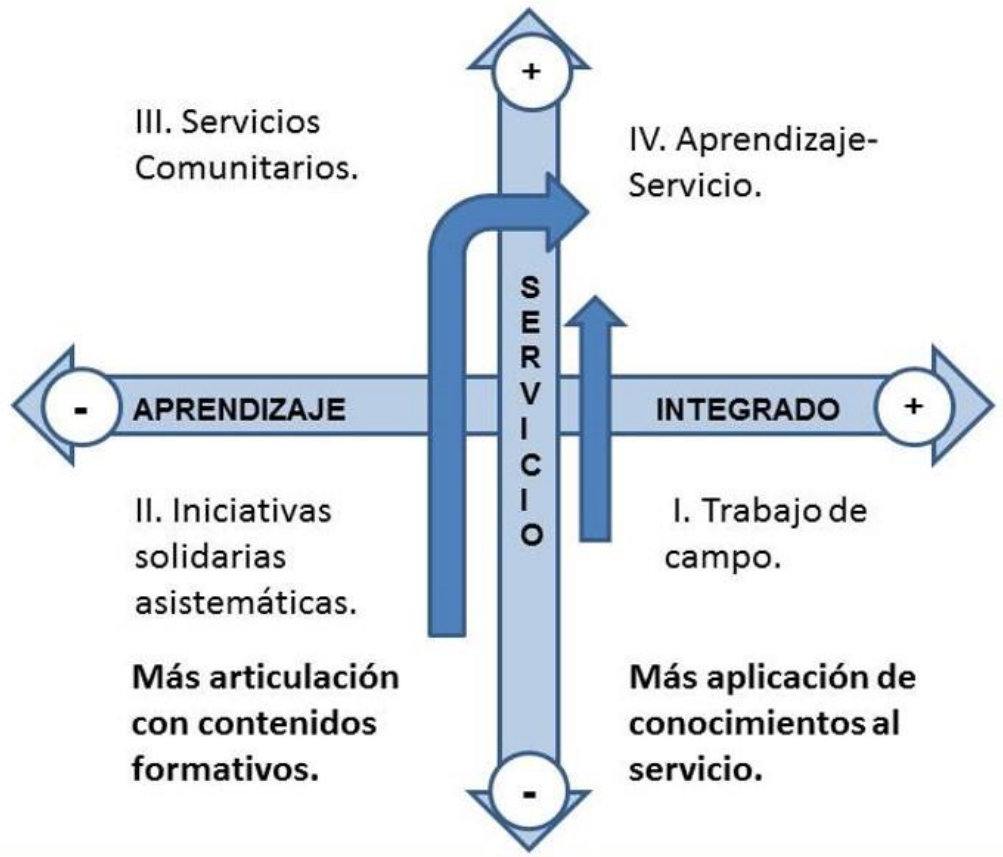

Fuente: Elaboración propia a partir de Montes, Tapia y Yaber (2011, p. 15 y p. 19) 
Para hacer posible el cambio hacia ApS "se hace necesario el análisis de cada una de las prácticas y orientarlas intencionalmente hacia un equilibrio entre los aprendizajes curriculares o formativos y las actividades que configuran el servicio a la comunidad" (Furco, 2011, p. 25).

\section{ApS como actividad de utilidad social}

Las prácticas de ApS, a diferencia de otras actividades formativas donde se pone el énfasis en el aprendizaje memorístico de contenidos curriculares sin valor de uso, introduce la acción en/con la comunidad con el propósito de mejorarla. Esta diferencia sustantiva tiene su idea germinal en el principio pedagógico planteado por Dewey (1924, p. 31): "actividad asociada con proyección social".

Con dicha expresión se quiere significar, por un lado, la necesidad que la educación parta de la experiencia real de sus protagonistas $y$, por otro, que dicha actividad no se cierre sobre sí misma, sino que se haga en provecho del entorno social que acoge a los participantes ya que, como planteaba Dewey (1985), solo implicándose en el perfeccionamiento del orden social se logrará la plena integración en la sociedad de cada nueva generación.

Lo expuesto anteriormente remite a la novedad que aporta el ApS en relación con otras experiencias educativas: "la planificación simultánea de la intencionalidad pedagógica y la intencionalidad social, mejorando así la calidad de los aprendizajes en tanto se articula teoría y práctica y planifica una respuesta participativa a una necesidad social" (Montes, Tapia y Yaber, 2011, p. 42).

\section{Los aprendizajes conectados al servicio}

El ApS constituye una actividad compleja que quiebra la forma de enseñanza de los dispositivos escolares tradicionales, basada en aprendizajes declarativos abstractos y descontextualizados, con escasa relevancia social. Esta forma tradicional de enseñar se traduce en aprendizajes poco significativos, relevantes o útiles, y en la incapacidad de los alumnos para transferir y generalizar lo que aprenden a situaciones de la vida real (Díaz, 2003; Puig, 2015; Rubio, 2007, Pérez, 2012).

En las actividades de ApS los aprendizajes, al estar imbricados con el servicio, favorecen la comprensión problemática de la realidad donde se va a actuar: diagnosticar y analizar las necesidades sociales a las que se pretende dar respuesta; implementar un servicio adecuado a dichas necesidades y reflexionar sobre lo acontecido. "Todo ello favorece la significatividad, relevancia y utilidad de los aprendizajes, ya que son puestos en acción para clarificar y afrontar problemas básicos de la comunidad y para ampliar horizontes de conocimientos, sensibilidades y afectos" (Pérez, 2012, p. 43).

En este sentido, lo que se aprende en las acciones de ApS se caracteriza por ser contextual, ya que parte de situaciones reales; por ser vivencial, puesto que requiere la 
implicación directa de los participantes en las distintas fases del proyecto; y por ser integral, en cuanto promueve el desarrollo de competencias necesarias para pensar y actuar sobre las realidades donde se ha decidido incidir.

Las competencias implican un saber pensar, decir, hacer y un querer relacionado con las necesidades e intereses y con el propio proyecto vital en contextos y situaciones concretas y complejas en función de propósitos deseados (Pérez, 2012). Constituyen el entramado básico, integrado por conocimientos, habilidades, actitudes y valores que utilizan los individuos para comprender e intervenir en los distintos ámbitos de la vida personal, social y profesional. A diferencia de los aprendizajes tradicionales, esta modalidad requiere, para su puesta en acción, abordar tareas complejas y desafíos que inciten a los participantes a movilizar sus recursos y a construir otros para intentar dar respuestas a las nuevas situaciones problemáticas que afronta.

En el contexto educativo, el ApS se puede implementar en el ámbito de la educación formal (desde infantil a universitaria), así como en el ámbito de la educación no formal, puesto que puede mejorar los procesos de aprendizaje al conectarlos a situaciones de la vida real que invitan a la reflexión-acción y permite el desarrollo de competencias para la vida atendiendo a las diferentes dimensiones de los individuos y grupos humanos.

A partir de los trabajos de Rubio (2007), hemos analizado las competencias que se pueden desarrollar en los proyectos de ApS que se implementan en el ámbito universitario (Tabla 1). 
Tabla 1. Competencias y aprendizajes en el aprendizaje-servicio

\begin{tabular}{|l|l|}
\hline $\begin{array}{l}\text { Competencias personales: orientadas a la } \\
\text { adquisición de aprendizajes para actuar de forma } \\
\text { autónoma y responsable. }\end{array}$ & $\begin{array}{l}\text { Autoconocimiento, autonomía, } \\
\text { compromiso y responsabilidad, } \\
\text { liderazgo, etc. }\end{array}$ \\
\hline $\begin{array}{l}\text { Competencias interpersonales: promueven } \\
\text { aprendizajes necesarios para aprender a vivir en } \\
\text { sociedad. }\end{array}$ & $\begin{array}{l}\text { Comunicación y expresión, diálogo, } \\
\text { resolución de conflictos, etc. }\end{array}$ \\
\hline $\begin{array}{l}\text { Competencias para el pensamiento reflexivo, } \\
\text { creativo y crítico: favorecen aprendizajes } \\
\text { orientados a comprender y valorar la complejidad } \\
\text { del medio social y su desarrollo en este. }\end{array}$ & $\begin{array}{l}\text { Curiosidad y motivación ante la realidad } \\
\text { compleja y cambiante, comprensión de } \\
\text { retos y problemas sociales, etc. }\end{array}$ \\
\hline $\begin{array}{l}\text { Competencias para la realización de proyectos: } \\
\text { posibilitan la adquisición de herramientas para } \\
\text { diseñar, implementar y evaluar acciones de Aps. }\end{array}$ & $\begin{array}{l}\text { Imaginación y creatividad para } \\
\text { planificar proyectos, trabajo en equipo, } \\
\text { difusión y transferencias de ideas y } \\
\text { proyectos, etc. }\end{array}$ \\
\hline $\begin{array}{l}\text { Competencias para la ciudadanía y la } \\
\text { transformación social: impulsa el logro de } \\
\text { aprendizajes para participar en defensa de los } \\
\text { bienes comunes como ciudadanos responsables. }\end{array}$ & $\begin{array}{l}\text { Comprensión de cuestiones sociales y } \\
\text { acciones políticas, participación } \\
\text { responsable en la comunidad, etc. }\end{array}$ \\
\hline $\begin{array}{l}\text { Competencias vocacionales y profesionales: } \\
\text { aportan aprendizajes y capacitación para el } \\
\text { desempeño en el mundo del trabajo. }\end{array}$ & $\begin{array}{l}\text { Conciencia de las opciones } \\
\text { vocacionales, preparación para el } \\
\text { mundo laboral, etc. }\end{array}$ \\
\hline
\end{tabular}

Fuente: Elaboración propia (2018) a partir del trabajo de Rubio (2007)

La Tabla 1 refleja un escenario amplio de posibles competencias y aprendizajes que los protagonistas de las prácticas de ApS pueden adquirir. Habrá que planificarlos y adaptarlos en función de las capacidades iniciales de los individuos y grupos, los objetivos del proyecto, el contexto donde se implementan y los avatares del proceso, sin perder de vista que una de las potencialidades pedagógicas del trabajo educativo por competencias reside en la necesidad de considerar todos los elementos que la conforman (conocimientos, habilidades, actitudes, valores), ya que "actúan dentro de un sistema que les da sentido, y sin el cual no puede entenderse la complejidad ni de la comprensión, ni de la actuación humana" (Pérez, 2012, p. 147).

\section{El servicio como respuesta a las necesidades sociales}

El vocablo servicio puede ser nombrado para significar cosas diferentes: labor o trabajo que se hace sirviendo a alguna entidad pública o privada; organización y personal destinados a satisfacer necesidades de la población; favor en beneficio de alguien; utilidad y provecho de algún objeto o actividad, entre otras. En todas las definiciones 
subyace la idea de cosa útil, necesaria o que interesa por algún motivo, pero carecen del potencial formador y transformador que adquieren en el marco de los proyectos de ApS, donde se entiende como una acción educativa planificada orientada a dar respuesta a necesidades reales de la sociedad (Puig y Palos, 2006).

Se trata de una actividad de utilidad social que es indisociable, como ya se ha señalado, de los aprendizajes que la originan y sustentan, así como de la intencionalidad transformadora que la orienta. Desde esta mirada, el servicio se encuentra en las antípodas de las actividades puramente caritativas o asistenciales que hace de quien recibe la asistencia un sujeto pasivo, sin posibilidad de participar en el proceso de mejora, para situarse en las proximidades del planteamiento de Freire (1983), quien concibe el conocimiento de la realidad como eje imbricado en las acciones transformativas, poniendo de relieve las dimensiones políticas, sociales y éticas de las acciones humanas.

En línea con lo anterior, las actividades de servicio se constituyen como acciones que los sujetos realizan de forma real y que, por tanto, se distancian de los debates teóricos sin aplicación práctica, la expresión de deseos o la mera especulación de posibles actuaciones. Un servicio es una experiencia práctica que, según Puig et al. (2007), Puig (2015) y Batlle (2009), debe estar articulada sobre las siguientes dimensiones:

- Emerge a partir de la deliberación, compromiso y organización de un plan establecido.

- Es una acción libre, consciente y gratuita que favorece la adquisición de aprendizajes múltiples y el desarrollo de competencias ciudadanas.

- Es una actividad orientada a mejorar algunos aspectos de la realidad social.

- Las acciones han de ser útiles y significativas para el conjunto de los protagonistas.

- Han de crear una estructura de reciprocidad en la que quien realiza el servicio también reciba algún beneficio que le aporta el destinatario de la actividad. Ello favorece una dinámica de promoción mutua.

En algunas ocasiones se detectan dudas para identificar las distintas calidades del servicio: integración de los aprendizajes, nivel de participación de los protagonistas, mejoras producidas en la comunidad, sostenibilidad de las acciones, entre otras. En esta línea, se está avanzando en los últimos años para intentar establecer criterios de calidad para evaluar los servicios. Una propuesta que se empieza a difundir en el escenario internacional es la elaboradora por Montes et al. (2011), configurada por criterios relacionados con:

- Duración y frecuencia de las acciones.

- Impactos positivos producidos en el aula, centro, entidad social, institución pública y comunidad donde se implementa el servicio.

- Nivel de satisfacción del conjunto de los participantes. 
- Posibilidad de alcanzar objetivos de cambio social a medio y largo plazo, a partir de las acciones emprendidas.

- Creación de redes interinstitucionales entre organizaciones sociales, instituciones públicas y empresas privadas.

- Sustentabilidad de las acciones realizadas.

Por otro lado, la variedad de servicios, el grado de integración en el currículum formativo, el foco del impacto, las edades de los participantes, los contextos socioculturales (Furco, 2011) dificultan la elaboración de una tipología cerrada que recoja la heterogeneidad y riqueza de los servicios implementados. Un acercamiento a este tema es el propuesto por Puig (2015) y Batlle (2009), que plantean una tipología general siguiendo como criterio la temática que orienta los servicios: Acompañamiento a la formación; Información, Asesoramiento y Acompañamiento; Relación intergeneracional; Preservación del medio ambiente y del patrimonio culturas; Participación ciudadana; Solidaridad y cooperación; Promoción de la salud. El modelo presentado no agota el ámbito del servicio, es solo un recorte parcial de este, un esfuerzo razonado para sistematizar esta práctica compleja que es el ApS.

\section{El aprendizaje-servicio como método}

En el universo educativo se vienen implementado diversas actividades orientadas a conseguir diferentes objetivos. Estas se regulan por disímiles métodos o conjuntos de procedimientos que se aplican para resolver problemas de toda índole, desde los cotidianos a los científicos. Estos pasos o fases se pueden resumir en: definición de retos, generación de ideas, diseño del plan de acción, implementación de acciones y evaluación. El ApS, como método educativo, mantiene una estrecha relación con algunos métodos de enseñanza-aprendizaje ampliamente conocidos, como el trabajo por proyectos, el estudio de casos y la solución de problemas (Díaz, 2006).

El trabajo por proyectos incluye tareas formales asignadas a individuos o grupos de alumnos relacionados con un área de estudio determinada.

Supone una representación que anticipa una intención de actuar o hacer alguna cosa, la elaboración de una perspectiva lo más amplia posible sobre el asunto de interés, así como la previsión prospectiva de las acciones necesarias para intervenir en la dirección pensada (Hernández y Ventura, 2008, p. 15).

Su implementación requiere que el alumnado investigue, cree o analice información que coincida con los objetivos del proyecto. Una de las fortalezas pedagógicas del trabajo por proyectos es la posibilidad de preparar al alumno no solo en torno a la experiencia concreta en que este participa, sino en la posibilidad de transferir lo aprendido en otros contextos. 
El método de aprendizaje basado en la solución de problemas se concibe como una estrategia de enseñanza-aprendizaje consistente en plantearle al alumnado situaciones reales o simuladas que se conectan con la aplicación de un ámbito de conocimiento o ejercicio profesional. En estas situaciones el alumnado tiene que analizar la situación y elegir una o varias alternativas viables de solución. Suele definirse como una experiencia educativa de tipo práctico, orientada a la investigación y resolución de problemas relacionados al mundo real, lo cual favorece el aprendizaje activo y la integración del aprendizaje escolar con la vida real (Díaz, 2003).

La metodología basada en el estudio de casos es considerada por algunos autores como una variante particular del método de resolución de problemas. Según Wassermann (citado en Díaz, 2006, p. 76):

Los casos son instrumentos educativos complejos que aparecen en forma de narrativas. Un caso incluye información y datos (psicológicos, históricos, económicos, etc.), así como material técnico. Aunque los casos se centran en materias o áreas curriculares específicas, son por naturaleza interdisciplinarios.

La utilización del análisis de casos con fines educativos favorece la capacidad de discutir con argumentos, de generar y sustentar ideas propias, de tomar decisiones en condiciones de incertidumbre o de realizar juicios de valor, sin dejar de lado el punto de vista de los demás y mostrar una actitud de apertura y tolerancia hacia las ideas de los otros (Díaz, 2003).

El ApS se diferencia de los anteriores métodos en tres cuestiones fundamentales: la conexión de los aprendizajes curriculares o formativos con las actividades que configuran el servicio a la comunidad; el hecho de que la finalidad educativa que lo orienta persigue la mejora de una situación social real y la participación fuerte del alumnado en las distintas fases de la actividad educativa.

Como estrategia metodológica se configura a partir de distintos dinamismos o elementos pedagógicos que se retroalimentan mutuamente. De los doce dinamismos identificados por Rubio, Puig, Martín y Palos (2015) en la rúbrica para la autoevaluación de experiencias de aprendizaje servicio, ${ }^{3}$ hemos considerado la participación, la reflexión, la investigación y la acción como las que configuran el anclaje metodológico. A continuación, se analizan algunas dimensiones pedagógicas que orientan la metodología de los proyectos de ApS: participación, investigación, acción y reflexión (Figura 4).

\footnotetext{
${ }^{3}$ La rúbrica recoge doce dinamismos pedagógicos agrupados en tres apartados: básicos, pedagógicos y organizativos Cada dinamismo tiene distintos niveles que manifiestan su grado de desarrollo pedagógico (Rubio et al., 2015).
} 
Figura 4. Dimensiones pedagógicas del aprendizaje-servicio como método educativo

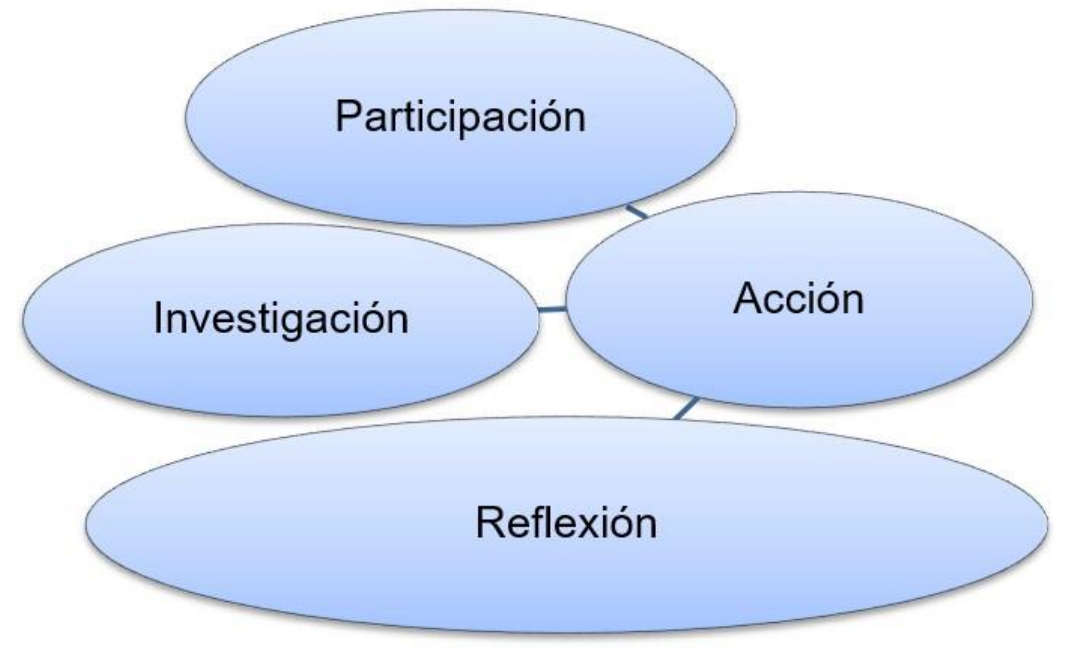

Fuente: Elaboración propia (2018)

La participación puede ser concebida como un constructo borroso que regula y se manifiesta de diferentes formas en distintos ámbitos del vivir humano en las sociedades democráticas. En el universo educativo, el tomar parte puede interpretarse de distintos modos: como derecho, objetivo, proceso, medio, contexto. De esta forma se manifiesta la diversidad de finalidades que persiguen las acciones que se implementan en los ámbitos de la educación formal y no formal.

En el ámbito de la educación escolar, se viene utilizando, en general, como un mecanismo burocrático al servicio de la gestión. En este sentido, Feito (2011, p.112) plantea que "todos los estudios que han analizado la participación del alumnado refieren una situación que oscila entre la sumisión y la indiferencia".

Asimismo, se emplea, en el ámbito del aula, para describir situaciones que conllevan desiguales niveles de implicación por parte del alumnado: escuchar, opinar, gestionar algo, estar apuntado a o ser miembro de, tomar decisiones. Ello pone de relieve la existencia de múltiples formas, tipos, grados y ámbitos de participación (Trilla y Novella, 2001).

Para clarificar dichos asuntos, se vienen desarrollando variados análisis que señalan las características de los diversos tipos de participación y los diferentes grados de implicación que conllevan (Tabla 2). 
Tabla 2. Distintos niveles de participación de los y las menores según diversos autores

\begin{tabular}{|l|l|l|l|l|}
\hline Hart (1993) & $\begin{array}{l}\text { Manipulación, } \\
\text { decoración y } \\
\text { simbolismo }\end{array}$ & $\begin{array}{l}\text { Informados } \\
\text { Consultados }\end{array}$ & $\begin{array}{l}\text { Inician adultos y } \\
\text { comparten con } \\
\text { menores }\end{array}$ & $\begin{array}{l}\text { Decisiones } \\
\text { compartidas } \\
\text { con los adultos }\end{array}$ \\
\hline Casas (1995) & Informados & Escuchados & $\begin{array}{l}\text { Participan en las } \\
\text { decisiones }\end{array}$ & $\begin{array}{l}\text { Se respetan las } \\
\text { decisiones }\end{array}$ \\
\hline Shier (2001) & Escuchados & Consultados & $\begin{array}{l}\text { Involucrados en la } \\
\text { toma de decisiones }\end{array}$ & $\begin{array}{l}\text { Decisiones } \\
\text { compartidas }\end{array}$ \\
\hline Trilla y Novella (2001) & $\begin{array}{l}\text { Participación } \\
\text { simple }\end{array}$ & $\begin{array}{l}\text { Participación } \\
\text { consultiva }\end{array}$ & $\begin{array}{l}\text { Participación } \\
\text { proyectiva }\end{array}$ & Metaparticipación \\
\hline Fielding (2011) & Fuente de información & Agentes & Coinvestigadores & Creadores de saber \\
\hline Rubio et al. (2015) & Cerrada & Delimitada & Compartida & Liderada \\
\hline
\end{tabular}

Fuente: Elaboración propia (2018) a partir de los trabajos de Rubio et al. (2015) y Trilla y Novella (2001)

De los modelos de participación expuestos, conviene señalar algunas cuestiones:

1) Los diversos tipos de participación reflejan, por un lado, maneras de participar cualitativa y cuantitativamente distintas, ya que favorecen desiguales niveles de implicación, así como la puesta en acción de distintas capacidades cognitivas, afectivas, comportamentales y relacionales. Por otro lado, evidencian la concepción de educación, alumnado o participantes y de práctica educativa que tiene la institución o personas encargadas de llevar a cabo la acción.

2) Las formas de participación no son excluyentes, ya que la participación cerrada o débil puede ser necesaria, en un primer momento, para el progreso hacia formas de participación más complejas, fuertes o compartidas. En este sentido, es importante identificar cómo y para qué se ponen en marcha los procesos participativos y el grado de protagonismo que asumen los participantes, ya que puede ser un elemento que sustenta modelos de educación cívica radicalmente diferentes (Susinos y Ceballos, 2012). 
En cuanto a la participación en los proyectos de ApS, se piensa desde una dimensión fuerte y negociada debido a que es una de sus señas de identidad junto a la vinculación de los aprendizajes y el servicio a la comunidad con el propósito de mejorarla. En este sentido, el ApS se postula como "una pedagogía de lo significativo y de lo que abre nuevas posibilidades" (Puig et al., 2007, p. 63), donde los participantes se involucran en asuntos públicos, asumiendo la responsabilidad que de ellos se deriva en las distintas etapas del proyecto: desde la elección del tema sobre el que se trabajará hasta la implementación de las acciones planificadas y la evaluación de la experiencia.

Además, la participación es planteada como un proceso orientado a la adquisición de aprendizajes múltiples y multidireccionales, ya que las prácticas de ApS se articulan como un escenario complejo donde confluyen distintos elementos (grupo de iguales, relaciones con personas de la comunidad, actividades en el medio real, aprendizajes orientados a la acción, reflexión de las experiencias). Todo ello favorece que los protagonistas de la experiencia vayan desarrollando sus capacidades cognitivas, afectivas, comportamentales y relacionales.

La investigación -considerada como una actividad sistemática dirigida a obtener mediante diversas técnicas de recogida de datos (observación, análisis de documentos, entrevistas, encuestas), informaciones y conocimientos relevantes sobre distintos aspectos de la realidad - se concibe como un elemento mediador de las prácticas de ApS que posibilita la extracción de datos para la configuración del diagnóstico inicial, donde quedan explicitadas las necesidades sociales detectadas en contextos próximos y singulares.

El análisis y la comprensión de las necesidades sociales favorece en los participantes, por un lado, el desarrollo de la capacidad crítica y el compromiso social y, por otro, la implicación en la construcción de acciones reflexivas que buscan mejorar algún aspecto de la realidad. Estudiar las necesidades sociales intentando analizar sus causas, identificar sus características y sus límites, se convierte en una herramienta poderosa para la construcción de conocimientos (Díaz, 2006) y eje impulsor del proceso de aprendizaje necesario para la realización del servicio. También favorece el desarrollo de los protagonistas como agentes sociales con responsabilidad en los asuntos públicos.

La acción, en el marco del ApS, es entendida como una actividad compleja que promueve la conexión de dimensiones sustantivas: la teoría con la práctica, la reflexión con la acción, la cognición con la emoción, el aula con la realidad y la formación con el compromiso social (Manzano, 2012). Son dimensiones que aparecen separadas en las instituciones escolares que siguen ubicadas en un enfoque tradicional de enseñanza y aprendizaje.

Como dinamismo pedagógico, la acción se puede situar en la intersección entre dos postulados de la pedagogía contemporánea: el aprendizaje por la acción o aprender haciendo (Dewey, 1985) y la acción transformadora (Freire, 1983). En esta línea de 
pensamiento, las actividades que la configuran están orientadas a promover aprendizajes significativos y útiles, dirigidos a mejorar aspectos de la realidad.

Por otra parte, en la articulación flexible de la acción pedagógica se integran dos dimensiones, la participación fuerte de los participantes y la reflexión, que funcionan como vasos comunicantes que retroalimentan el proceso de planificación de las actividades a implementar (Mayor y Rodríguez, 2016). Desde esta mirada la acción, que se materializa en el servicio a la comunidad o actividad de utilidad social, intenta evitar caer en el activismo acrítico que reproduce las condiciones sociales. Para ello organiza espacios que favorecen el revolverse hacia dentro o reflexión, con la intencionalidad de posibilitar, en los protagonistas, la creación de sentido personal y social.

La reflexión es concebida como una cualidad humana que favorece la optimización del aprendizaje a través de un proceso de análisis, creación de relaciones e identificaciones, a través de la cual el sujeto se vuelve y se revuelve sobre los objetos percibidos o conocidos para profundizar y mejorar la propia experiencia individual y grupal. Como plantea Dewey (1985), se trata de volver sobre la experiencia para examinarla con mayor atención e incrementar el conocimiento que ha de servir para una mayor comprensión.

Asimismo, la reflexión en las acciones de ApS se entiende como un proceso sistemático que se aleja de la reflexión de bajo perfil u ocasional que suele acompañar a toda acción humana, previsto en "distintos momentos destinados a ejercerla, así como de estrategias para facilitarla" (Páez y Puig, 2013, p. 16). Ello implica generar espacios para valorar, cuestionar, analizar, criticar y tomar conciencia de la experiencia, lo que posibilita que los participantes puedan dotar de significado personal y social a la acción que están realizando.

Siguiendo a Deeley (2016), pueden destacarse como aportes de la reflexión a las prácticas de ApS los siguientes:

- Los procesos reflexivos se convierten en revulsivos contra el activismo que puede suponer el servicio, pero también contra el academicismo en el que, con frecuencia, derivan los aprendizajes descontextualizados.

- El revolverse hacia dentro potencia la conexión de las dos dimensiones sustantivas que constituyen las prácticas de ApS: los aprendizajes y el servicio a la comunidad.

- La reflexión recorre las diversas etapas del proyecto (planificación, implementación y evaluación) y se configura como un eje transversal, con el propósito de que los participantes puedan construir los sentidos de su implicación. 


\section{Agentes y relaciones para el aprendizaje-servicio}

El interés por construir puentes que favorezcan la relación escuela-comunidad en orden de aprovechar los recursos educativos diseminados en el medio es una necesidad que vienen planteando en sus obras autores reconocidos en el marco de la pedagogía contemporánea como Dewey (1985) y Freire (1983), así como estudiosos del ámbito educativo (Carbonell, 2014; Delors ,1996).

Por otra parte, desde hace algunas décadas, las políticas sociales implementadas en el ámbito europeo vienen apostando por la organización de actuaciones integrales constituidas por planes, programas y proyectos que favorezcan el tratamiento global de las problemáticas detectadas en materia de educación, vivienda, salud y empleo en los distintos territorios, con el propósito de promover sinergias entre los distintos recursos públicos y privados que trabajan en un mismo escenario.

La necesidad de desarrollar políticas y prácticas, tanto en el ámbito social como en el educativo, sustentadas en el trabajo coordinado, colaborativo o asociativo, es un asunto recurrente en sus diseños y una propuesta de mejora en relación con las políticas y prácticas tradicionales con marcado carácter sectorial y paternalista. En esta línea, se vienen implementando prácticas sociales y educativas (Programa de Naciones Unidas para el Desarrollo, Planes de Desarrollo Local o Comunitario, Ciudades Educadoras, Comunidades de Aprendizaje, etc.) con el propósito de promover lazos fuertes 0 relaciones de partenariado entre los diversos actores que trabajan en el territorio.

Igualmente, los proyectos de ApS requieren por naturaleza la conexión entre variados dispositivos que, aunque inicialmente tienen fines, características e intereses desiguales, pueden articular acciones con objetivos comunes que potencian la capacidad educativa de cada uno de ellos.

\section{CONCLUSIONES}

A lo largo del texto nos hemos adentrado en las dimensiones nucleares que configuran las prácticas pedagógicas de ApS: aprendizajes conectados al servicio a la comunidad; acción educativa orientada a mejorar aspectos de la realidad; participación fuerte de las personas implicadas en los distintos momentos del proyecto; la reflexión como eje transversal que recorre el vivir de la experiencia y el trabajo colaborativo entre agentes y agencias educativas. Estos aspectos sirven para identificar sus señas de identidad y las similitudes y diferencias con otras prácticas pedagógicas experienciales y otros métodos educativos.

En este sentido se puede concluir que, a la luz de la literatura revisada, las prácticas de ApS se diferencian de otras prácticas educativas experienciales por constituirse en el entramado de las dimensiones pedagógicas y las sociales. Un aspecto diferencial que sirve para dotar de sentido a los contenidos objeto de estudio y focalizar la atención educativa en el conocimiento como valor de uso. 
En cuanto a su relación con otros métodos educativos (aprendizaje basado en proyectos; aprendizaje basado en problemas, entre otros), se pueden apreciar, en general, diferencias significativas en las finalidades que los guían, la conexión circular de los contenidos curriculares y las actividades derivadas del servicio a la comunidad, así como la necesidad de establecer alianzas con otros agentes educativos para su implementación.

Aunque cada practica de ApS se configura como un microuniverso dependiente del contexto y circunstancias que lo rodean, se puede concluir, después de revisar numerosas publicaciones en el ámbito iberoamericano, que los proyectos de ApS contienen virtualidades pedagógicas para ser considerados como contextos apropiados para promover el desarrollo de competencias en los distintos niveles educativos (Butin, 2006; Rodríguez, 2014; Rubio, 2007; Rubio et al., 2015). Esto se debe a que el alumnado se tiene que enfrentar a situaciones problemáticas de la vida real que tendrá que analizar y conectar a los aprendizajes curriculares para construir soluciones provisionales que mejoren la situación planteada.

El ApS también se ha mostrado como una herramienta metodológica pertinente para promover la corresponsabilidad de los distintos agentes educativos insertos en el territorio (Batlle, 2013; Mayor y Rodríguez, 2016), puesto que los proyectos están orientados a mejorar aspectos de la realidad social donde otros agentes y agencias educativas ya están trabajando o se plantean iniciar una intervención con la intencionalidad de optimizarla. En definitiva, el ApS se configura como una práctica experiencial que favorece la adquisición de valores, conocimientos y herramientas para la formación de una ciudadanía activa, reflexiva y crítica (Carbonell, 2014; Puig y Palos, 2006; Puig et al., 2007).

\section{REFERENCIAS}

Blanchard, M., y Muzás, M. A. (2016). Los proyectos de aprendizaje. Un marco metodológico clave para la innovación. Madrid, España: Narcea.

Batlle, R. (2009). El servicio en el aprendizaje servicio. En J. M. Puig (coord.), Aprendizaje servicio. Educación y compromiso cívico (pp. 71-90). Barcelona, España: Graó.

Batlle, R. (2010). Aprendizaje-servicio y entidades sociales. Aula de Innovación Educativa, 192, 66-68.

Batlle, R. (2013). El aprendizaje-servicio en España: el contagio de una revolución pedagógica necesaria. Madrid, España: PPC.

Butin, D. W. (2006). The limits of service-learning in higher education. The Review of Higher Education, 29 , 473-498. Recuperado de

http://citeseerx.ist.psu.edu/viewdoc/download?doi=10.1.1.392.9924\&rep=rep1\&type=pdf Carbonell, J. (2014). Pedagogías del S. XXI. Alternativas para la innovación educativa. Barcelona, España:

Octaedro. 
Deeley, S. (2016). El aprendizaje-servicio en la educación superior. Teoría, práctica y perspectiva crítica. Madrid, España: Narcea.

Delors, J. (Coord.). (1996). La educación encierra un tesoro. Madrid, España: Santillana.

Dewey, J. (1924). Ensayos de educación. Madrid, España: La Lectura.

Dewey, J. (1985). Democracia y escuela. Barcelona, España: Eumo.

Díaz, F. (2003). Cognición situada y estrategias de aprendizaje significativo. Revista Electrónica de Investigación Educativa, 5(2), 1-13.

Díaz, F. (2006). Enseñanza situada: vínculos entre la escuela y la vida. México D. F., México: McGraw-Hill Interamericana.

Feito, R. (2011). Los retos de la participación escolar: elección, control y gestión de los centros educativos. Madrid, España: Morata.

Freire, P. (1983). La educación como práctica de la libertad. Madrid, España: Siglo XXI.

Furco, A. (2011). El aprendizaje-servicio: un enfoque equilibrado de la educación experiencial. Revista Educación Global, 0, 64-70.

Hernández, F., y Ventura, M. (2008). La organización del currículum por proyectos de trabajo. Barcelona, España: Octaedro.

Luna, E. (2010). Del centro educativo a la comunidad: un programa de aprendizaje-servicio para el desarrollo de ciudadanía activa (Tesis Doctoral). Universitat de Barcelona, España. Recuperado de http://www.tdx.cat/bitstream/handle/10803/81944/ELG_TESIS.pdf?sequence=1

Manzano, V. (2012). La Universidad comprometida. Bilbao, España: Hegoa.

Martínez-Odria, A. (2008). Service-learning o aprendizaje-servicio. La apertura de la escuela a la comunidad local como propuesta de educación para la ciudadanía. Bordón, 59(4), 627-640.

Mayor, D., y Rodríguez, D. (2016). Aprendizaje-Servicio y práctica docente: una relación para el cambio educativo. Revista de Investigación Educativa, 32(2), 535-552. https:// doi.org/10.6018/rie.34.2.231401

Montes, R., Tapia, M., y Yaber, L. (2011). Manual para docentes y estudiantes solidarios. Buenos Aires, Argentina: CLAYSS. Recuperado de http://www.clayss.org.ar/04_publicaciones/Natura2013.pdf

Páez, M., y Puig, J. M. (2013). La reflexión en el aprendizaje-servicio. Revista Internacional de Educación para la Justicia Social, 2(2), 13-32. Recuperado de http://www.rinace.net/riejs/numeros/vol2num2/art1.pdf

Pérez, A. I. (2012). Educarse en la era digital. Madrid,, España: Morata.

Puig, J. M. (Coord.). (2009). Aprendizaje Servicio. Educación y compromiso cívico. Barcelona, España: Graó.

Puig, J. M. (Coord.). (2015). 11 ideas clave. ¿Cómo realizar un proyecto de aprendizaje-servicio? Barcelona, España: Graó.

Puig, J. M., Batlle, R., Bosch, C., y Palos, J. (2007). Aprendizaje servicio. Educar para la ciudadanía. Madrid, España: Octaedro. 
Puig, J. M., y Palos, J. (2006). Rasgos pedagógicos del aprendizaje-servicio. Cuadernos de Pedagogía, 357, 60-63.

Rodríguez, M. (2014). Aprendizaje-Servicio como estrategia metodológica en la Universidad. Revista Complutense de Educación, 25(1), 95-113. Recuperado de http://revistas.ucm.es/index.php/RCED/article/view/41157

Rubio, L. (2007). El aprendizaje en el aprendizaje servicio. En J. M. Puig (Coord.), Aprendizaje servicio. Educación y compromiso cívico (pp. 91-105). Barcelona, España: Graó.

Rubio, L., Puig, J. M., Martín, X., y Palos, J. (2015). Analizar, repensar y mejorar los proyectos: una rúbrica para la autoevaluación de experiencias de aprendizaje servicio. Revista de currículum y formación del profesorado, 1(19), 111-126. Recuperado de http://www.ugr.es/ recfpro/rev191ART6.pdf

Susinos, T., y Ceballos, N. (2012). Voz del alumnado y presencia participativa en la vida escolar. Apuntes para una cartografía de la voz del alumnado en la mejora educativa. Revista de Educación, 359, 24-44. https:// doi.org/10.4438/1988-592X-RE-2012-359-194

Tapia, M. N., González, A., y Elicegui, O. (2005). Aprendizaje y Servicio Solidario en las escuelas argentinas: Una visión descriptiva a partir de las experiencias presentadas al Premio Presidencial Escuelas Solidarias (2000-2001). Buenos Aires, Argentina: CLAYSS.

Trilla, J., y Novella, A. M. (2011). Educación y participación social de la infancia. Revista Iberoamericana de Educación, 26, 137-164. Recuperado de http://diposit.ub.edu/dspace/bitstream/2445/56924/1/502327.pdf 